\title{
MAN.08 - The role of technological roadmaps in shaping the future strategy for Bio-Manguinhos
}

Lethícia Mallet ${ }^{1 *}$; Rosângela Alves ${ }^{1}$; Monique Menegaci ${ }^{1}$; Pedro Barbosa ${ }^{2}$; Elaine Vasconcelos do Nascimento Gomes ${ }^{1}$.

1Fiocruz/Bio-Manguinhos;

2IBMP- Instituto de Biologia Molecular do Paraná.

Introduction: The development of technological roadmaps has been a widely used technique in organizations to help map and manage the strategic portfolio that meet future market challenges. In particular, for organizations with high technological intensity, such as the pharmaceutical and biotechnology, roadmaps can help in the construction of articulated visions between demands - defined by technological and market trends - and the internal possibilities and resources of achieving them. Considering the review of Bio-Manguinhos' Strategic Plan (2017-2028), Planning and Organization Advisory proposed the adoption of roadmapping technique to reliably support the executive board on analysis and selection of priority technological paths in the business areas of the Institute.

Objective: The objective is to present how the roadmapping methodology was applied and adapted for Bio-Manguinhos to support the strategic reflections for the next ten years, highlighting the major contributions to Strategic Plan results obtained by each business segment - Vaccines, Biopharmaceuticals and Diagnostics.

Methodology: The methodology was an adaptation of the roadmapping technique adopted at the Institute of Manufacturing of the University of Cambridge, in which the key dynamics is based on articulation between market-product-technology-resources. Three roadmaps were built, for each product segment, adopting the Strategic Planning horizon - 10 years. First of all, Planning Advisory organized data collection to subsidize workshops for experts discussion. It was based on market studies and identification of technological trends (prospective analysis) prepared by Marketing and Technological internal teams. These studies were held at the beginning of three workshops organized by segment to support specialists filling in the maps with priority and viable routes for Bio-Manguinhos, considering short, medium and long term horizons.

Results: The application of the technique resulted in three strategic roadmaps based on a set of technological paths considered by the specialists as institutional priorities, which were reference to Board decision-making and selection of the strategic drivers to be pursued in each business segment. Among different drivers, we highlight some examples - the importance of investing in the synthetic biology as a future route for vaccines; pursuing the development of immunoconjugate products and personalized medicine in biopharmaceuticals segment; and invest in the new generation of molecular tests based on the point-of-care platform, in Diagnostics.

Conclusion: The adoption of roadmapping technique allows discussing perspectives and visions of the future in the light of a set of resources that must be mobilized to develop products that meet the markets of interest of the business. In addition, it supports qualified board decisions about the future reflected in the set of strategic drivers that should guide the entry of new products and the priority operations for the Institute in the short, medium and long term.

Keywords: roadmapping; strategy; technology 